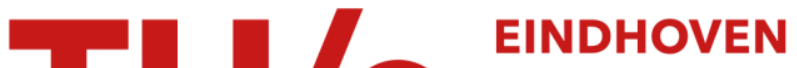 UNIVERSITY OF TECHNOLOGY
}

\section{Flexible model structures for LPV identification with static scheduling dependency}

\section{Citation for published version (APA):}

Toth, R., Heuberger, P. S. C., \& Hof, Van den, P. M. J. (2008). Flexible model structures for LPV identification with static scheduling dependency. In Proceedings of the 47th IEEE Conference on Decision and Control (CDC 2008), 9-11 December 2008, Cancun, Mexico (pp. 4522-4527). Institute of Electrical and Electronics Engineers. https://doi.org/10.1109/CDC.2008.4739028

DOI:

10.1109/CDC.2008.4739028

Document status and date:

Published: 01/01/2008

\section{Document Version:}

Accepted manuscript including changes made at the peer-review stage

\section{Please check the document version of this publication:}

- A submitted manuscript is the version of the article upon submission and before peer-review. There can be important differences between the submitted version and the official published version of record. People interested in the research are advised to contact the author for the final version of the publication, or visit the $\mathrm{DOI}$ to the publisher's website.

- The final author version and the galley proof are versions of the publication after peer review.

- The final published version features the final layout of the paper including the volume, issue and page numbers.

Link to publication

\section{General rights}

Copyright and moral rights for the publications made accessible in the public portal are retained by the authors and/or other copyright owners and it is a condition of accessing publications that users recognise and abide by the legal requirements associated with these rights.

- Users may download and print one copy of any publication from the public portal for the purpose of private study or research.

- You may not further distribute the material or use it for any profit-making activity or commercial gain

- You may freely distribute the URL identifying the publication in the public portal.

If the publication is distributed under the terms of Article 25fa of the Dutch Copyright Act, indicated by the "Taverne" license above, please follow below link for the End User Agreement:

www.tue.nl/taverne

Take down policy

If you believe that this document breaches copyright please contact us at:

openaccess@tue.nl

providing details and we will investigate your claim. 


\title{
Flexible model structures for LPV identification with static scheduling dependency
}

\author{
R. Tóth, P. S. C Heuberger, and P. M. J Van den Hof
}

\begin{abstract}
A discrete-time Linear Parameter-Varying (LPV) model can be seen as the combination of local LTI models together with a scheduling signal dependent function set, that selects one of the models to describe the continuation of the signal trajectories at every time instant. An identification strategy of LPV models is proposed that consists of the separate approximation of the local model set and the scheduling functions. The local model set is represented as a linear combination (series expansion) of Orthonormal Basis Functions (OBFs). The expansion coefficients are dynamically dependent (weighting) functions of the scheduling parameters (depending on time shifted scheduling). To approximate this dependency class with a static one (non-shifted scheduling), a feedbackbased structure of the weighting functions is introduced. The proposed model structure is identified in a two step procedure. First the OBFs, that guarantee the least asymptotic worst-case modeling error for the local models, are selected through the Fuzzy Kolmogorov $c$-Max approach. With the resulting OBFs, the weighting functions are identified through a separable leastsquares algorithm. The method is demonstrated by means of simulation examples and analyzed in terms of applicability, convergence, and consistency of the model estimates.
\end{abstract}

Index Terms - orthonormal basis, identification, LPV.

\section{INTRODUCTION}

Many physical/chemical processes exhibit parameter variations due to non-stationary or nonlinear behavior or dependence on external variables. For such processes, the theory of Linear Parameter-Varying (LPV) systems offers an attractive modeling framework [1]. LPV systems are generally described in either a State-Space (SS) or an Input/Output (IO) representation [2], where the signal relations are considered to be linear just as in the Linear Time-Invariant (LTI) framework, but the model parameters are assumed to be functions of a measurable time-varying signal, the so-called scheduling variable $p: \mathbb{Z} \rightarrow \mathbb{P}$. The compact set $\mathbb{P} \subset \mathbb{R}^{n_{\mathbb{P}}}$ denotes the scheduling space. Due to the parameter variation, the LPV system class can describe both time-varying and nonlinear phenomena. Practical use of this framework is stimulated by the fact that LPV control design is well worked out, extending results of optimal and robust LTI control theory to nonlinear, time-varying plants [1], [3], [4]. However, it still remains a problem how to develop/identify LPV models of physical processes in a systematic fashion.

Recently several methods have been proposed, aiming at global identification of discrete-time LPV models from

Support by the Netherlands Organization for Scientific Research (NWO) is gratefully acknowledged. The authors are with the Delft Center for Systems and Control, Delft University of Technology, Mekelweg 2, 2628 CD, Delft, The Netherlands, email: r.toth@tudelft.nl. measured data [5], [6], [7], [8], [9], [10]. Most approaches exploit the fact that an LPV system $\mathcal{S}$ can be viewed as a collection of "local" models connected by scheduling dependent weighting functions [1], [11]. For a constant scheduling signal: $p(k)=\overline{\mathrm{p}} \forall k \in \mathbb{Z}$, the LPV system $\mathcal{S}$ is identical to an LTI system $\mathcal{F}_{\bar{p}}$. Thus, the set of local models of $\mathcal{S}$ is given as $\mathfrak{F}_{\mathbb{P}}=\left\{\mathcal{F}_{\overline{\mathrm{p}}}\right\}_{\overline{\mathrm{p}} \in \mathbb{P}}$. The $p$-dependent weighting function set, that schedules on $\mathfrak{F}_{\mathbb{P}}$, is denoted by $\mathfrak{H}_{\mathbb{P}}=\left\{h_{\overline{\mathrm{p}}}(.)\right\}_{\overline{\mathrm{p}} \in \mathbb{P}}$. An efficient way of LPV system identification can lead through the separate identification of $\mathfrak{F}_{\mathbb{P}}$ and $\mathfrak{H}_{\mathbb{P}}$.

Identification of $\mathfrak{F}_{\mathbb{P}}$ is commonly accomplished in a 'sampled' sense, by a number of LTI identification experiments of $\mathcal{S}$ for a set of constant scheduling signals. Then, assuming that the scheduling functions $\left\{h_{\overline{\mathrm{p}}}\right\}$ have a particular structure of dependency, for instance polynomial, an interpolation problem is formulated on $\mathbb{P}$ to obtain a global approximation of $\mathcal{S}$. Recent research showed that this approach should be handled with care [2], [11], [12]. In [2] it was shown that for general LPV systems, each $h_{\overline{\mathrm{p}}}$ is a function of time-shifted versions of $p$ (dynamic dependency). Then, if a too simple interpolation structure of $\left\{h_{\overline{\mathrm{p}}}\right\}$ is chosen (static dependency, linear dependency, etc.), the interpolation based on statespace or IO model parametrization can result in significantly different models [2]. An additional concern of interpolation is that the McMillan degree of the local systems $\left\{\mathcal{F}_{\bar{p}}\right\}$ may vary for different values of $\bar{p} \in \mathbb{P}$. This shows that the choice of a model structure which can incorporate aspects of dynamical dependency and local order changes is crucial for this identification approach.

The Orthonormal Basis Functions (OBFs)-based models offer such a structure with a well worked-out theory in the context of LTI system approximation and identification [13]. These functions, that provide bases for the system space $\mathcal{H}_{2}$, are generated by a cascaded network of stable all-pass filters, whose poles represent the prior knowledge about the system at hand. This approach characterizes the transfer function of a strictly proper LTI system as

$$
F(z)=\sum_{i=1}^{\infty} w_{i} \phi_{i}(z)
$$

where $\left\{w_{i}\right\}_{i=1}^{\infty}$ is the set of coefficients and $\Phi_{\infty}=\left\{\phi_{i}\right\}_{i=1}^{\infty}$ represents the sequence of OBFs. Consequently, every $\mathcal{F}_{\bar{p}} \in$ $\mathfrak{F}_{\mathbb{P}}$ can be represented as a linear combination of a given $\Phi_{\infty}$, i.e. $\mathfrak{F}_{\mathbb{P}} \subset \operatorname{span}\left\{\Phi_{\infty}\right\}$. In practice, only a finite number of terms is used in (1), like in Finite Impulse Response (FIR) models. In contrast with FIR structures, the OBF 
parametrization can achieve almost zero modeling error with a relatively small number of parameters, due to the infinite impulse response characteristics of the basis [13]. In this way, it is generally possible to find a finite $\Phi_{n} \subset \Phi_{\infty}$, with a relatively low number of functions $n \in \mathbb{N}$, such that the representation error for all $\mathcal{F}_{\overline{\mathrm{p}}}$ is negligible. If a data record is used where $p$ is a varying signal, then by assuming any linearly parameterized functional dependence, like polynomial, of the scheduling function on the timeshifted versions of $p$, an interpolation can be formulated with dynamical $p$-dependency. In this way, global identification of general LPV systems becomes available. Furthermore, local order changes do not affect this parametrization [11], [14].

Two problems remain to be solved with the proposed LPV identification approach. The first is to choose an OBF set $\Phi_{n}$, "sufficiently rich" to describe $\mathfrak{F}_{\mathbb{P}}$. In [15] a FuzzyKolmogorov $c$-Max $(\mathrm{FK} c \mathrm{M})$ approach was proposed to solve this selection problem through the fusion of Kolmogorov $n$ width theory for OBFs [16] and Fuzzy c-Means clustering [17] of sample poles of $\mathfrak{F}_{\mathbb{P}}$. This approach guarantees in an asymptotic $^{1}$ sense the least worst-case local modeling error for $\mathfrak{F}_{\mathbb{P}}$ with the resulting OBFs [15], [14].

The second problem is that - in order to enable representation of the general LPV class - the weighting functions need rational dynamic dependence on the scheduling parameters (for a detailed discussion see [12]). The identification of such functions is a difficult problem, as in practical situations the required order of time shifts is unknown. This results in an extra freedom of the model structure. In [11] the OBFs model structure with static dependency was considered. This structure can represent LPV-SS systems where only the $\{B, D\}$ or the $\{C, D\}$ matrices of an SS realization $\{A, B, C, D\}$ depend on $p$ in a static way, as well as LPV-IO systems where the autoregressive part has a particular dependency structure. In this paper, we aim at the approximation of dynamic dependencies, using a feedback-based structure of weighting functions with static dependency. It is shown that this weighting structure can approximate a wide class of dynamical weighting functions, enabling better approximation of most LPV systems, while retaining the merits of static dependency.

The paper is organized as follows: Section II describes an OBFs based model structure and its properties for LPV system approximation; in Section III feedback based weighting functions are introduced for the approximation of dynamic dependencies while in Section IV identification of the proposed model structure is described; in Section V applicability of the approach is shown through an example; and in Section VI, the main results of the paper are discussed.

\section{LPV OBF MODEL STRUCTURES}

As stated, OBFs based identification has attractive properties in the LTI case. To use them in the identification of general LPV systems, we introduce a model structure, that

\footnotetext{
${ }^{1}$ In terms of $m$, the fuzziness parameter of the clustering algorithm. If $m \rightarrow \infty$, optimality is guaranteed (see [15]).
}

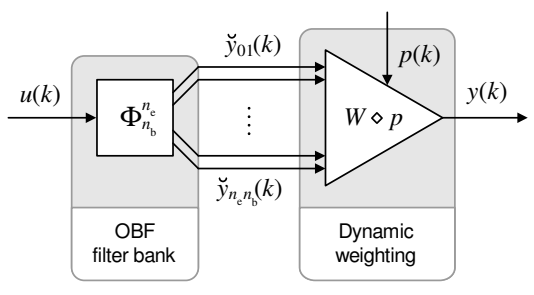

Fig. 1. IO signal flow graph of the W-LPV OBF model described by (4), with $W=\left[\begin{array}{lll}w_{01} & \ldots & w_{n_{e}, n_{b}}\end{array}\right]$.

consists of an OBF filter bank, connected to a weighting function set with dynamic dependence on $p$. For reasons of clarity we describe the model structure for the SISO case. See Figure 1 for a schematic overview. In this figure the symbol $\diamond$ is used to describe the evaluation of the function $W$ along $p$ in a dynamic sense, as explained in more detail later in this section. The structure has some resemblance with nonlinear Wiener models ${ }^{2}$, important model classes for chemical, biological, and sensor/actuator systems [18]. A fundamental difference with the classical Wiener models is the dynamic nature of the output weighting (see [11]). In the sequel the introduced structure is called a Wiener LPV OBF model (W-LPV OBF) and its signal relations are analyzed and the LPV system representation capabilities are investigated. Let $\mathcal{H}_{2-}(\mathbb{E})$ denote the Hardy space of strictly proper functions, analytic on $\mathbb{E}$, the exterior of the unit disk, and squared integrable on the unit circle. In the following, we consider the Hambo class of OBFs in $\mathcal{H}_{2-}(\mathbb{E})$ defined as follows [13]. Let $G_{b}$ be an inner function with McMillan degree $n_{b}>1$ and minimal balanced SS representation $\left\{A_{b}, B_{b}, C_{b}, D_{b}\right\}$. Denote by $e_{j}$ the $j^{t h}$ standard basis vector of $\mathbb{R}^{n_{b}}$, and let

$$
\phi_{j}(z)=e_{j}^{T}\left(z I-A_{b}\right)^{-1} B_{b} .
$$

Then the Hambo OBFs, defined as

$$
\Phi_{n_{b}}^{n_{e}}=\left\{\phi_{j}(z) G_{b}^{i}\right\}_{j=1, \cdots, n_{b}}^{i=0, \cdots, n_{e}},
$$

with $n_{e}=\infty$ constitute a basis for $\mathcal{H}_{2-}(\mathbb{E})$. Furthermore, let $\mathcal{R}$ be the field of real meromorphic ${ }^{3}$ functions dependent on variables $\left\{\zeta_{i j}\right\}_{i=-n_{\zeta}, \ldots, n_{\zeta}}^{j=1, \ldots, n_{\mathbb{P}}}$ with $n_{\mathbb{P}}, n_{\zeta} \in \mathbb{N}$. Associate each variable $\zeta_{i j}$ with

$$
\zeta_{i j}=q^{i} p_{j}
$$

where $q$ denotes the forward time-shift operator and $p_{j}$ is the $j^{t h}$ element of $p$ with $\mathbb{P} \subseteq \mathbb{R}^{n_{\mathbb{P}}}$.

Introduce the operator $\diamond$, such that for a given function $w \in \mathcal{R}$ with variables $\left\{\zeta_{i j}\right\}_{i=-n_{\zeta}, \ldots, n_{\zeta}}^{j=1, \ldots, n_{\mathbb{P}}}$ and a scheduling signal $p: \mathbb{Z} \rightarrow \mathbb{P}$ :

$$
(w \diamond p)(k)=w\left(\left\{p_{j}(k+i)\right\}_{i=-n_{\zeta}, \ldots, n_{\zeta}}^{j=1, \ldots, n_{\mathbb{P}}}\right) .
$$

In this way $w$ can be used to represent a weighting function with dynamic dependency on $p$. Then, the IO form of the

\footnotetext{
${ }^{2}$ LTI models with static output nonlinearity are called Wiener models.

${ }^{3} \mathrm{~A}$ real meromorphic function $f: \mathbb{R}^{n} \rightarrow \mathbb{R}$ is a function that is differentiable except in a set of isolated points [19].
} 
W-LPV OBF model (Figure 1) of a SISO LPV system $\mathcal{S}$ is:

$$
y=\sum_{i=0}^{n_{e}} \sum_{j=1}^{n_{b}}\left(w_{i j} \diamond p\right) \underbrace{\phi_{j}(q) G_{b}^{i}(q) u}_{\breve{y}_{i j}} .
$$

If $\mathfrak{F}_{\mathbb{P}} \subseteq \operatorname{span}\left\{\Phi_{n_{b}}^{n_{e}}\right\}$, then every $\mathcal{F}_{\overline{\mathrm{p}}} \in \mathfrak{F}_{\mathbb{P}}$ is realizable by the W-LPV OBF model and the $p$-dependent transient behavior of $\mathcal{S}$ is contained in $\left\{w_{i j}\right\}$. For this property, generally infinitely many functions, $n_{e}=\infty$, are required, so using a finite number of basis functions restricts the class of realizable LPV systems. In practice however, careful selection of the basis can ensure almost error free representation of $\mathfrak{F}_{\mathbb{P}}$ with a limited number of OBFs (see [15]).

Furthermore, if $\mathfrak{F}_{\mathbb{P}} \subseteq \operatorname{span}\left\{\Phi_{n_{b}}^{n_{e}}\right\}$ holds, then based on [2] and [20], it can be shown that the introduced model structures can completely represent any general LPV system $\mathcal{S}$ (either IO or SS). In this way, (4) can be viewed as a series expansion of the LPV system in terms of the LTI bases with parameter dependent expansion coefficients. As a series expansion, the W-LPV OBF model is also well structured against changes of the local McMillan degree of $\mathcal{S}$. This shows that the introduced model parameterization is not affected by problems that are common for other LPV model parameterizations (see Section I).

\section{APPROXIMATION OF DYNAMIC DEPENDENCY}

The main purpose of the OBFs based model structure is to give a flexible representation that is able to handle general LPV systems and which is unaffected by local changes of the system order. In order to describe any LPV system, the weighting functions need to have dynamic dependency on the scheduling signal $p$, which is an extra freedom of the model structure. This means that in a practical identification setting, a wrong choice of the functional dependency can easily lead to over-parametrization or significant bias. To overcome this problem, approximation with static dependency was suggested in [11] for W-LPV OBF models, with the drawback that this limits the class of representable LPV systems. In this paper, we propose to still use weighting functions with static dependency, but with the introduction of an additional feedback loop around each basis component with a gain incorporating also static dependency (see Figure 2). In this way, the filter bank of OBFs as a dynamical LTI system is "reused" to provide dynamic output or input weighting functions that can approximate the required class of dependency for W-LPV OBF models. The introduction of feedback-based weighting leads to a new model structure given in Figure 2, which we call Wiener Feedback (WF) LPV models.

Denote the input and output of each basis function in $\Phi_{n_{b}}^{n_{e}}$ by $\breve{u}_{i j}$ and $\breve{y}_{i j}$ satisfying:

$$
\breve{y}_{i j}=\phi_{j}(q) G_{b}^{i}(q) \breve{u}_{i j} .
$$

Let $\mathcal{R}_{n_{\mathbb{P}}}$ denote the field of real meromorphic functions with variables $\left\{\zeta_{0 j}\right\}_{j=1}^{n_{\mathbb{P}}}$ where each variable $\zeta_{0 j}$ is associated with the $j^{t h}$ element of $p$ (without time-shift). So for $w \in$ $\mathcal{R}_{n_{\mathbb{P}}}$ the notation $w \diamond p$ represents a weighting function with

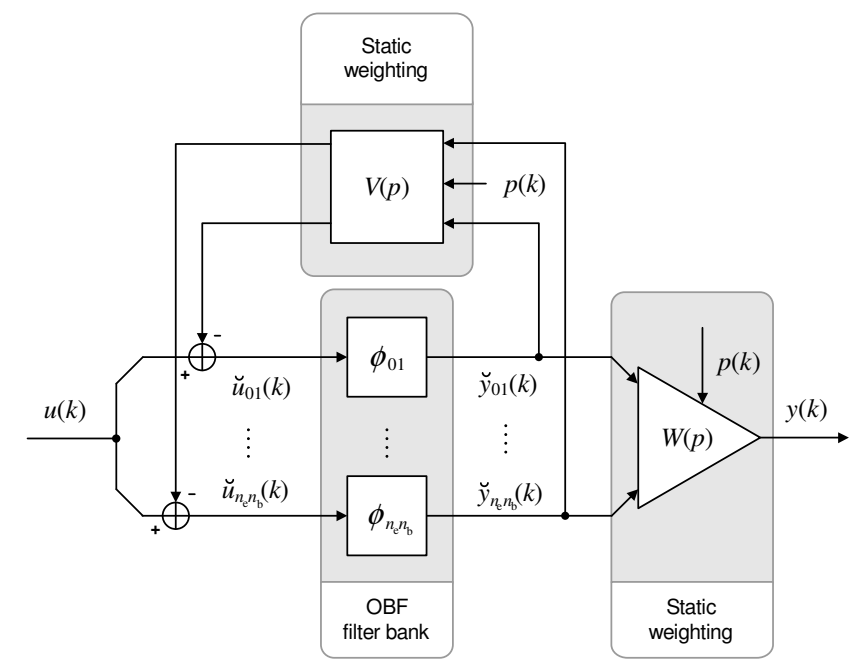

Fig. 2. IO signal flow graph of W-LPV OBF models with feedback based static weighting functions $V$ and $W$.

static dependency on $p$. To distinguish static from dynamic dependency, we denote by $w(p)$ the evaluation of a static $w \in \mathcal{R}_{n_{\mathbb{P}}}$ along the scheduling trajectory $p$. Then for the approximation of a SISO LPV system $\mathcal{S}$, the WF-LPV OBF model is given as

$$
\begin{aligned}
\breve{u}_{i j} & =u-v_{i j}(p) \breve{y}_{i j}, \\
y & =\sum_{i=0}^{n_{e}} \sum_{j=1}^{n_{b}} w_{i j}(p) \breve{y}_{i j},
\end{aligned}
$$

where each $\breve{u}_{i j}$ and $\breve{y}_{i j}$ satisfies (5) additionally. Let $\left\{A_{i j}, B_{i j}, C_{i j}\right\}$ be a minimal balanced SS realization of $\phi_{j} G_{b}^{i}$ and introduce $A_{b}=\operatorname{diag}\left(A_{01}, \ldots, A_{n_{e} n_{b}}\right)$ and define $B_{b}$, and $C_{b}$ accordingly. Denote $W(p)=\operatorname{diag}\left(w_{01}(p), \ldots\right.$, $\left.w_{n_{e} n_{b}}(p)\right)$ and $V(p)$ accordingly and let $E_{b}=\left[\begin{array}{lll}1 & \ldots & 1\end{array}\right]$. Then, the SS equivalent of (5) and (6a-b) is given as

$$
\begin{aligned}
q x_{b} & \left.=\left[A_{b}-B_{b} V(p) C_{b}\right)\right] x_{b}+B_{b} E_{b}^{T} u \\
y & =W(p) C_{b} x_{b} .
\end{aligned}
$$

Again, if $\mathfrak{F}_{\mathbb{P}} \subseteq \operatorname{span}\left\{\Phi_{n_{b}}^{n_{e}}\right\}$, then every $\mathcal{F}_{\overline{\mathrm{p}}} \in \mathfrak{F}_{\mathbb{P}}$ is realizable by the WF-LPV model and the $p$-dependent transient behavior of $\mathcal{S}$ is approximated by $\left\{w_{i j}, v_{i j}\right\}$. The following theorem has a main importance:

Theorem 3.1: For any uniformly stable WF-LPV OBF model, with OBF set $\Phi_{n_{b}}^{n_{e}}$ and static weighting functions $w_{i j}, v_{i j} \in \mathcal{R}_{n_{\mathbb{P}}}$, there exists a W-LPV OBF model associated with dynamic weighting functions, such that both models are equivalent in the IO sense.

See [12] for a proof of this theorem. The converse of Theorem 3.1 does not hold in general. Hence, a WF-LPV OBF model can generally only approximate an LPV system $\mathcal{S}$. However it is able to approximate the dynamic weighting function class of the W-LPV equivalent representation of $\mathcal{S}$, through its feedback-based static weighting functions $w_{i j}, v_{i j} \in \mathcal{R}_{n_{\mathbb{P}}}$. Clearly such an approximation is more adequate than a static approximation without feedback (each $v_{i j}=0$ ) which was applied in [11]. Equations (7a-b) 
imply that the WF-LPV structure can approximate parameter dependence in the $A$ matrix as well as parameter dependence in the autoregressive part, see (6a-b), of LPV-SS and LPVIO models. It has wider representation capabilities than the W-LPV OBF model structure with static dependency, which is a special case of the WF-LPV structure. However, this improved representation capability comes at a price, namely that due to the feedback, stability of the model is not internally guaranteed like in the W-LPV case. This also yields that the equivalent W-LPV OBF model in terms of Theorem 3.1 does not necessarily have the same basis. These aspects, as we will see, can be crucial in identification of LPV systems with the WF-LPV OBF structure.

\section{LPV IDENTIFICATION}

In the following, an approach is proposed for identification using the WF-LPV OBF model structure. As mentioned in Section I, a key problem of OBFs based identification is the choice of the basis functions $\Phi_{n_{b}}^{n_{e}}$. For completeness, first a practically applicable basis selection mechanism based on [15] is briefly discussed. Then, assuming that a set of basis functions is given, the WF-LPV identification approach based on a separable least squares algorithm is presented.

\section{A. OBF selection}

Assume that a set of scheduling points $\mathcal{P}=\left\{\overline{\mathrm{p}}_{i}\right\}_{i=1}^{N_{p}} \subset \mathbb{P}$ is given for a uniformly stable $\mathcal{S}$, where it is expected that $\mathfrak{F}_{\mathcal{P}}=\left\{\mathcal{F}_{\overline{\mathrm{p}}_{i}}\right\}_{i=1}^{N_{p}}$ contains representative samples of the dynamics of all systems in $\mathfrak{F}_{\mathbb{P}}$. Then the basis selection procedure is as follows:

1) Determination of pole samples of the pole functionals of $\mathcal{S}$ by LTI identification of each $\mathcal{F}_{\overline{\mathrm{p}}} \in \mathfrak{F}_{\mathcal{P}}$.

2) Determination of the optimal OBF set $\Phi_{n_{b}}^{n_{e}}$ for $\mathcal{S}$ based on $\mathrm{FK} c \mathrm{M}$ clustering [15]. The procedure also copes with the uncertainty of the pole estimates.

This algorithm aims at optimality of the OBF selection with respect to any LTI system that has its poles in the clustered pole regions. The prior knowledge, i.e. the information about the possible local poles of $\mathcal{S}$, directly effects the optimality of the selection.

\section{B. Parameter estimation}

Opposite to the basis selection approach, the second step of the identification method utilizes only one data set $\mathcal{D}_{N_{d}}=\{y(k), u(k), p(k)\}_{k=0}^{N_{d}-1}$ which is collected from $\mathcal{S}$ with varying scheduling and it is assumed to be $\mathrm{PE}^{4}$ for $\mathcal{S}$. Assume a linearly parameterized functional dependence of $W(p)$ and $V(p)$, for instance that each $w_{i j}$ and $v_{i j}$ is a polynomial of the elements of $p$. To overcome the nonlinear optimization problem associated with the parallel estimation of the whole parameter set, the approach utilizes a separable least squares optimization scheme [22]. In each iteration cycle of this scheme, one set of the parameters is fixed to enable a linear-regression-based estimation of

\footnotetext{
${ }^{4} \mathrm{PE}$ stands for Persistently Exciting signals. Conditions are hard to be drawn for general LPV systems and they are subject of research. See [21].
}

the other set. This results in a steepest descend algorithm which is guaranteed to converge to a saddle point or a local minimum, depending on the initial values of the parameters. The procedure is given in detail as follows:

3) Parameterize each $w_{i j}$ and $v_{i j}$ of (6a-b) as

$$
\begin{aligned}
w_{i j}(p) & =\sum_{l=0}^{n_{\mathrm{v}}} r_{i j l}^{\mathrm{W}} \psi_{l}^{\mathrm{w}}(p), \\
v_{i j}(p) & =\sum_{l=0}^{n_{\mathrm{v}}} r_{i j l}^{\mathrm{v}} \psi_{l}^{\mathrm{v}}(p),
\end{aligned}
$$

where $\left\{r_{i j l}^{\mathrm{v}}\right\}$ and $\left\{r_{i j l}^{\mathrm{W}}\right\}$ are real coefficients and $\psi_{l}^{\mathrm{W}}, \psi_{l}^{\mathrm{v}} \in \mathcal{R}_{n_{\mathbb{P}}}$ are functional dependencies chosen by the user with $\psi_{0}^{\mathrm{w}}=\psi_{0}^{\mathrm{v}}=1$.

4) Choose an initial set of values for $\left\{r_{i j l}^{\mathrm{v}}\right\}$.

5) Use the data to compute $\breve{u}=\left[\breve{u}_{i j}\right]_{j=1 \ldots n_{b}}^{i=0, \ldots, n_{e}}$ and $\breve{y}=$ $\left[\breve{y}_{i j}\right]_{j=1 \ldots, \ldots n_{b}}^{i=0, \ldots, n_{e}}$ via (5) and (6a) with respect to the OBF set $\Phi_{n_{b}}^{n_{e}}$ resulting in Step 2.

6) Estimate the parameter set $\left\{r_{i j l}^{\mathrm{W}}\right\}$ by linear regression with respect to fixed $\left\{r_{i j l}^{\mathrm{v}}\right\}$. This is done by defining the regressors as

$$
\gamma^{T}(k)=\breve{y}(k) \otimes \psi^{\mathrm{w}}(p(k)),
$$

with $\otimes$ denoting the Kronecker tensor product and with $\psi^{\mathrm{w}}=\left[\begin{array}{lll}\psi_{0}^{\mathrm{w}} & \ldots & \psi_{n_{\mathrm{w}}}^{\mathrm{w}}\end{array}\right]$. Collect the data into $\Gamma_{N_{d}}=\left[\gamma(0) \ldots \gamma\left(N_{d}-1\right)\right]^{T}$ and $Y_{N_{d}}=$ $\left[y(0) \ldots y\left(N_{d}-1\right)\right]^{T}$. Organize the parameters to be estimated as $\Theta^{\mathrm{W}}=\left[\begin{array}{lllll}r_{010}^{\mathrm{W}} & \cdots & r_{01 n_{r}}^{\mathrm{W}} & \ldots & r_{n_{e} n_{b} n_{\mathrm{w}}}^{\mathrm{W}}\end{array}\right]^{T}$. Then to minimize the prediction error criterion:

$$
\mathcal{V}_{N_{d}}\left(\Theta^{\mathrm{w}}, \mathcal{D}_{N_{d}}\right)=\frac{1}{N_{d}}\left\|Y_{N_{d}}-\Gamma_{N_{d}} \Theta^{\mathrm{w}}\right\|_{2}^{2}
$$

the analytic solution is

$$
\breve{\Theta}^{\mathrm{w}}=\left[\frac{1}{N_{d}} \Gamma_{N_{d}}^{T} \Gamma_{N_{d}}\right]^{-1}\left[\frac{1}{N_{d}} \Gamma_{N_{d}}^{T} Y_{N_{z}}\right]
$$

7) Fix $\left\{r_{i j l}^{\mathrm{w}}\right\}$ at the values obtained in $\breve{\Theta}^{\mathrm{w}}$ and optimize the other parameters $\left\{r_{i j l}^{\mathrm{v}}\right\}$ in the following iterative way. In each iteration step we calculate an update for each element of $\Theta_{i j}^{\mathrm{v}}=\left[r_{i j 0}^{\mathrm{v}} \ldots r_{i j n_{\mathrm{v}}}^{\mathrm{v}}\right]$ in parallel and choose that element which gives the best improvement on the prediction error of the model:

a) For each $\phi_{i j}\left(=\phi_{j} G_{b}^{i}\right)$, compute

$$
\tilde{y}_{i j}=\frac{1}{w_{i j}(p)}\left[y-\sum_{k=0, k \neq i}^{n_{e}} \sum_{l=1, l \neq j}^{n_{b}} w_{k l}(p) \breve{y}_{k l}\right] .
$$

If $w_{i j}(p(k))=0$ for some $k$, then do not consider those time instants in the further procedure.

b) Collect each $\tilde{y}_{i j}$ into $\tilde{Y}_{N_{d}}^{(i j)}$ and $u$ into $U_{N_{d}}$ similarly to $Y_{N_{d}}$. Let $H_{i j}$ be the lower triangular Toeplitz matrix of the Markov parameters associated with $\left\{A_{i j}, B_{i j}, C_{i j}\right\}$. Define $\gamma_{i j}^{T}(k)=$ $\breve{y}_{i j}(k) \otimes \psi^{\mathrm{v}}(p(k))$ and collect it into $\Gamma_{N_{d}}^{(i j)}$. Then based on (7a-b) it holds that

$$
\tilde{Y}_{N_{d}}^{(i j)}=H_{i j} U_{N_{d}}-H_{i j} \Gamma_{N_{d}}^{(i j)} \Theta_{i j}^{\mathrm{v}}+\varepsilon_{i j},
$$

where $\varepsilon_{i j}$ is the error term. Then estimation of $\Theta_{i j}^{\mathrm{v}}$ can be formulated as a linear regression, similarly 


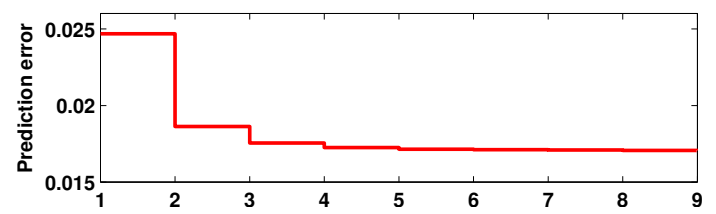

Fig. 3. Typical convergence plot of the prediction error for the iterative WF-LPV identification algorithm.

as in Step 6, with regressor $H_{i j} \Gamma_{N_{d}}^{(i j)}$ and data $H_{i j} U_{N_{d}}-\tilde{Y}_{N_{d}}^{(i j)}$.

c) For each element of $\Theta_{i j}^{\mathrm{v}}$ compute the prediction error of the model with only that element updated. Choose the element which renders the smallest error and only update the value of this element.

d) If the resulting prediction error did converge, stop, else goto Step 7a.

8) If the prediction error also converged with respect to both $\left\{r_{i j l}^{\mathrm{W}}\right\}$ and $\left\{r_{i j l}^{\mathrm{v}}\right\}$, then stop, else goto 6 .

Similar to the optimization schemes of LTI ARMAX or BoxJenkins models, the global optimum can only be obtained by starting the iterative search from different initial values and comparing the results [23]. A further problem may arise when the resulting model estimate is unstable even if $\mathcal{S}$ is uniformly stable. This phenomenon is due to the fact that the feedback weighting is tuned on a particular, finite scheduling trajectory $\left(\mathcal{D}_{N_{d}}\right)$. As this feedback tuning can be thought of as reoptimization of the basis with respect to $\mathcal{D}_{N_{d}}$, the finite data length and the excitation capabilities of the input and scheduling signals directly effect the estimation. Consequently also consistency of the model estimates is not guaranteed. However, as illustrated in Section V, the proposed method quickly converges in practice and provides a reliable estimate of LPV systems.

\section{EXAMPLE}

In this section, applicability of the introduced model structure is illustrated. Comparison is also made with the static weighting function based Wiener type of OBF model structure without feedback, introduced in [11]. An asymptotically stable LPV system $\mathcal{S}$ is considered, in LPV-IO form:

$$
\sum_{l=0}^{5} a_{l}(p(k)) y(k-l)=b_{1}(p(k)) u(k-1)
$$

where $p: \mathbb{Z} \rightarrow[0.6,0.8]$ and $a_{0}(p)=0.58-0.1 p$, $a_{1}(p)=-\frac{511}{860}-\frac{48}{215} p^{2}+0.3(\cos (p)-\sin (p)), a_{2}(p)=$ $\frac{61}{110}-0.2 \sin (p), a_{3}(p)=-\frac{23}{85}+0.2 \sin (p), a_{4}(p)=$ $\frac{12}{125}-0.1 \sin (p), a_{5}(p)=-0.003, b_{1}(p)=\cos (p)$. It can be shown (see [15]), that the dynamic changes of $\mathcal{S}$ are quite heavy between different constant scheduling points. Using sample poles of $\mathfrak{F}_{\mathbb{P}}$ associated with $\mathcal{P}=\{0.2+k \alpha\}_{k=0}^{10}$ and $\alpha=0.04$, the FKcM algorithm with fuzzyness $m=25$ resulted in a OBF set $\Phi_{7}^{0}$ with poles:

$$
\{0.183,0.147 \pm 0.28 i, 0.434 \pm 0.322 i, 0.352 \pm 0.478 i\} .
$$
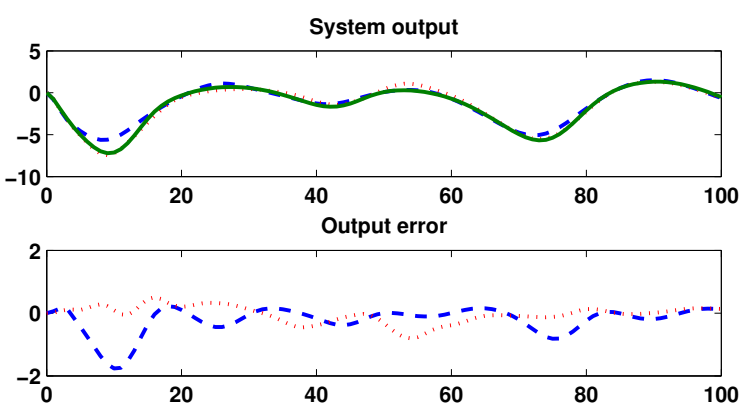

(a) multisine validation
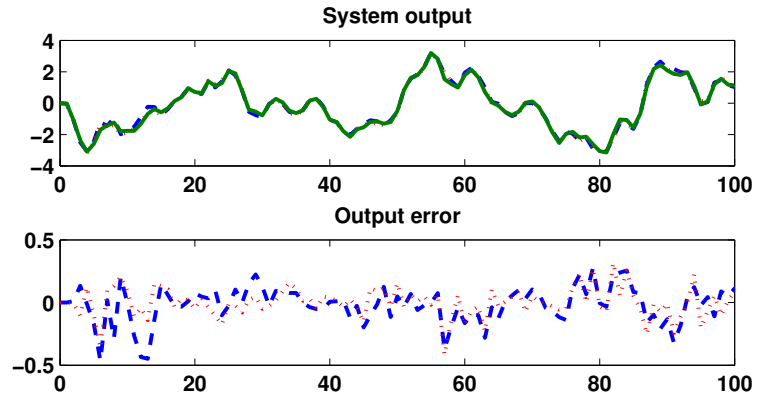

(b) uniform noise + multisine validation

Fig. 4. Typical validation results of identified WF-LPV OBF (dotted red) and W-LPV OBF (dashed blue) models in the SNR=20dB case. The response of the true system is given by solid green.

Identification of $\mathcal{S}$ was accomplished 100 times in 4 different noise settings with both model structures. The data was generated by excitation of $(u, p)$ based on uniform noise $(\mathcal{U}(-0.5,0.5), \mathcal{U}(0.65,0.75))$ superimposed on random multisines ( 3 sines with random phase and frequency and with overall amplitude of 0.5 and 0.05 ). For each data record, identification was accomplished in a noiseless setting and also with additive white output noise with variance $\sigma^{2}=0.01,0.1$, and 0.5. The resulting Signal to Noise Ratio (SNR) was $35 \mathrm{~dB}, 20 \mathrm{~dB}$, and $10 \mathrm{~dB}$, while the relative noise amplitude was $7 \%, 25 \%$, and $54 \%$ in average for the three noise cases. For the $\sigma^{2}=0.5$ case $N_{d}=1000$, and in the other cases $N_{d}=500$ sample long data records were used. The functional dependence on $p$ was chosen to be polynomial for both $W$ and $V$ and the feedback weights were initialized at zero. It was found that the optimal order for these polynomial parameterizations is 2 for $W$ and 3 for $V$ with these basis functions. The method converged in an average of 14 iterations for the $4 \times 100$ runs. A typical convergence plot is given in Figure 3 and the outputs of the resulting estimates are shown in Figure 4. In Table I, the (in)validation results are shown for deterministic multisine $(u, p)$ with random frequencies and phases and also for uniform noise superimposed on random multisine, similarly generated like the excitation in the data records. As expected, both approaches identified the system with 


\begin{tabular}{|c|c|c|c|c|c|c|c|c|c|c|c|c|}
\hline \multirow[b]{3}{*}{ SNR } & \multicolumn{6}{|c|}{ Validation by multisine } & \multicolumn{6}{|c|}{ Validation by uniform noise + multisine } \\
\hline & \multicolumn{2}{|c|}{ MSE (dB) } & \multicolumn{2}{|c|}{ BFT (\%) } & \multicolumn{2}{|c|}{ VAF (\%) } & \multicolumn{2}{|c|}{ MSE (dB) } & \multicolumn{2}{|c|}{ BFT (\%) } & \multicolumn{2}{|c|}{ VAF (\%) } \\
\hline & $\mathrm{W}$ & WF & $\mathrm{W}$ & WF & W & WF & $\mathrm{W}$ & WF & W & WF & $\mathrm{W}$ & WF \\
\hline no noise & -18.23 & -31.32 & 85.39 & 94.04 & 97.84 & 99.55 & -34.96 & -39.75 & 90.04 & 92.40 & 99.00 & 99.42 \\
\hline $35 \mathrm{~dB}$ & -18.21 & -31.30 & 85.38 & 93.64 & 97.82 & 99.53 & -34.77 & -39.17 & 89.92 & 92.15 & 98.99 & 99.39 \\
\hline $20 \mathrm{~dB}$ & -17.81 & -21.60 & 85.17 & 89.30 & 97.75 & 98.52 & -32.75 & -35.01 & 88.69 & 90.06 & 98.71 & 99.00 \\
\hline $10 \mathrm{~dB}$ & -20.68 & -22.60 & 86.27 & 88.44 & 98.47 & 98.98 & -31.81 & -32.38 & 87.73 & 88.15 & 98.19 & 98.59 \\
\hline
\end{tabular}

TABLE I

VALIDATION RESUlTS OF 100 IDENTIFICATION EXPERIMENTS WITH THE WIENER (W) AND THE WIENER FEEDBACK (WF) MODEL STRUCTURES.

adequate $\mathrm{MSE}^{5}, \mathrm{BFT}^{6}$, and $\mathrm{VAF}^{7}$ even in case of extremely heavy output noise, which underlines the effectiveness of the dual identification philosophy. For all measures, validation signals, and noise cases, the WF-LPV OBF model provided better estimates than the pure static dependency based WLPV model estimate. This clearly shows the improvement in the approximation capability. Additional extension of $\Phi_{7}$ with $n_{e}=1,2, \ldots$ did not improve the results as $\Phi_{7}$ is well chosen with respect to $\mathcal{S}$, i.e. the local modeling error is negligible due to the $\mathrm{FK} c \mathrm{M}$ [15]. Even in the $\mathrm{SNR}=10 \mathrm{~dB}$ case, the model estimates proved to be accurate, showing that the proposed identification scheme is applicable even in harsh white noise conditions.

\section{CONCLUSION}

In this paper, a feedback-based static weighting function structure was proposed for the approximation of the dynamic weighting functions of W-LPV representations of LPV systems. It was shown that such a weighting function structure provides good approximations for dynamic dependencies, by incorporating only a linear static parametrization over the scheduling. Identification of the proposed model structure was solved through a separable least squares approach. Through an example, it was justified that the proposed identification method provides fast and numerically stable model estimates even in case of strong output additive white noise. The presented approach provides an alternative for LPV system identification where the produced models give a close approximation of the original system and the method overcomes problems of interpolation, representation of statespace and IO models, and locally changing McMillan degree.

\section{REFERENCES}

[1] W. Rugh and J. Shamma, "Research on gain scheduling," Automatica, vol. 36, no. 10, pp. 1401-1425, 2000.

[2] R. Tóth, F. Felici, P. S. C. Heuberger, and P. M. J. Van den Hof, "Discrete time LPV I/O and state space representations, differences of behavior and pitfalls of interpolation," in Proc. of the European Control Conf., 2007, pp. 5418-5425.

[3] C. W. Scherer, "Mixed $H_{2} / H_{\infty}$ control for time-varying and linear parametrically-varying systems," Int. Journal of Robust and Nonlinear Control, vol. 6, no. 9-10, pp. 929-952, 1996.

\footnotetext{
${ }^{5}$ Mean Square Error, the expected value of the squared estimation error: $\mathrm{MSE}=\mathbb{E}\left(\left(y-y_{b}\right)^{2}\right)$.

${ }^{6}$ Best Fit percentage, the percentage of the output variation that is explained by the model. BFT $=100 \% \cdot \max \left(1-\frac{\left\|y-y_{b}\right\|_{2}}{\|y-\bar{y}\|_{2}}, 0\right)$ where $\bar{y}$ is the mean of $y$.

${ }^{7}$ Variance Accounted For percentage is defined as VAF $=100 \%$. $\max \left(1-\frac{\operatorname{var}\left(y-y_{b}\right)}{\operatorname{var}(y)}, 0\right)$ and computed on noise free $y$.
}

[4] K. Zhou and J. C. Doyle, Essentials of Robust Control. Prentice-Hall, 1998.

[5] L. Giarré, D. Bauso, P. Falugi, and B. Bamieh, "LPV model identification for gain scheduling control: An application to rotating stall and surge control problem," Control Engineering Practice, vol. 14, pp. 351-361, 2006.

[6] M. Sznaier, C. Mazzaro, and T. Inanc, "An LMI approach to control oriented identification of LPV systems," in Proc. of the American Control Conf., 2000, pp. 3682-3686.

[7] P. L. dos Santos, J. A. Ramos, and J. L. M. de Carvalho, "Identification of linear parameter varying systems using an iterative deterministicstochastic subspace approach," in Proc. of the European Control Conf., 2007, pp. 4867-4873.

[8] F. Felici, J. W. van Wingerden, and M. Verhaegen, "Subspace identification of MIMO LPV systems using a periodic scheduling sequence," Automatica, vol. 43, pp. 1684-1697, 2006.

[9] M. G. Wassink, M. van de Wal, C. Scherer, and O. Bosgra, "LPV control for a wafer stage: Beyond the theoretical solution," Control Engineering Practice, vol. 13, pp. 231-245, 2004.

[10] V. Verdult and M. Verhaegen, "Subspace identification of multivariable linear parameter-varying systems," Automatica, vol. 38, no. 5, pp. 805814, 2002.

[11] R. Tóth, P. S. C. Heuberger, and P. M. J. Van den Hof, "LPV system identification with globally fixed orthonormal basis functions," in Proc. of the 46th IEEE Conf. on Decision and Control, 2007, pp. 3646-3653.

[12] R. Tóth, "Modeling and identification of linear parameter-varying systems. An orthonormal basis function approach," Ph.D. dissertation, Delft University of Technology, 2008, to appear.

[13] P. S. C. Heuberger, P. M. J. Van den Hof, and Bo Wahlberg, Modeling and Identification with Rational Orthonormal Basis Functions. Springer-Verlag, 2005.

[14] R. Tóth, P. S. C. Heuberger, and P. M. J. Van den Hof, "Asymptotically optimal orthonormal basis functions for LPV system identification," Accepted for publication in Automatica.

[15] R. Tóth, P. S. C. Heuberger, and P. M. J. Van den Hof, "Orthonormal basis selection for LPV system identification, the Fuzzy-Kolmogorov c-Max approach," in Proc. of the 45th IEEE Conf. on Decision and Control, 2006, pp. 2529-2534.

[16] T. Oliveira e Silva, "A $n$-width result for the generalized orthonormal basis function model," in Preprints of the 13th IFAC World Congress, vol. I, 1996, pp. 375-380.

[17] J. C. Bezdek, Pattern Recognition with Fuzzy Objective Function Algorithms. Plenum Press, 1981.

[18] S. Billings and S. Fakhouri, "Identification of systems containing linear dynamic and static nonlinear elements," Automatica, vol. 18, no. 1, pp. 15-26, 1982.

[19] S. G. Krantz, Handbook of Complex Variables. Birkhäuser Verlag, 1999.

[20] S. Boyd and L. O. Chua, "Fading memory and the problem of approximating nonlinear operators with Volterra series," IEEE Trans. on Circuits and Systems, vol. 32, no. 11, pp. 1150-1161, 1985.

[21] X. Wei and L. Del Re, "On persistent excitation for parameter estimation of quasi-LPV systems and its application in modeling of diesel engine torque," in Proc. of the 14th IFAC Symposium on System Identification, 2006, pp. 517-522.

[22] G. H. Golub and V. Pereyra, "The differentiation of pseudo-inverses and nonlinear least squares problems whose varaibles are separate," SIAM Journal on Numerical Analysis, vol. 10, no. 2, pp. 413-432, 1973.

[23] L. Ljung, System Identification, theory for the user. Prentice Hall, 1999. 